To the Editors:

\title{
Wound infection survey at Teaching Hospital, Peradeniya
}

Despite many advances in surgery, wound infection continues to be a common problem $(1,2,3)$. It increases the morbidity and cost of patient care (4). Many hospitals have wound care teams and regular surveillance of wounds (5).

A prospective study was carried out from July to December 1997. A senior staff nurse was assigned to collect data from patient records before and after surgery. The grade of surgeon was recorded. The information recorded was kept confidential and during the period of studies the practice of surgery was not altered. Post-operatively all wounds were examined on the 3,7 and 30 day after surgery. Wound infection was defined as the presence of a discharge from the suture line with positive bacterial culture or the presence of frank pus which required drainage. The operations were classified according to the degree of contamination using guidelines given by the American College of Surgeons (5). Contaminated and dirty wounds were not included in this study.

We graded the operations into major, moderate and minor based on operative procedures. In this study, laparotomy, thoracotomy, thyroidectomy, mastectomy, and similar operations were graded as major. Less extensive operations such as hernia repair and appendicectomy were considered as moderate operations. Outpatient procedures and minor operations were excluded because of the difficulties in follow up of these patients. All traumatic wounds were excluded because many of them were left open and some were closed with systemic antibiotic therapy.

Over the 6-month period 211 patients were evaluated. Follow up was incomplete in another 52 patients and they were excluded. There were 30 wound infections. The Table summarises the results.

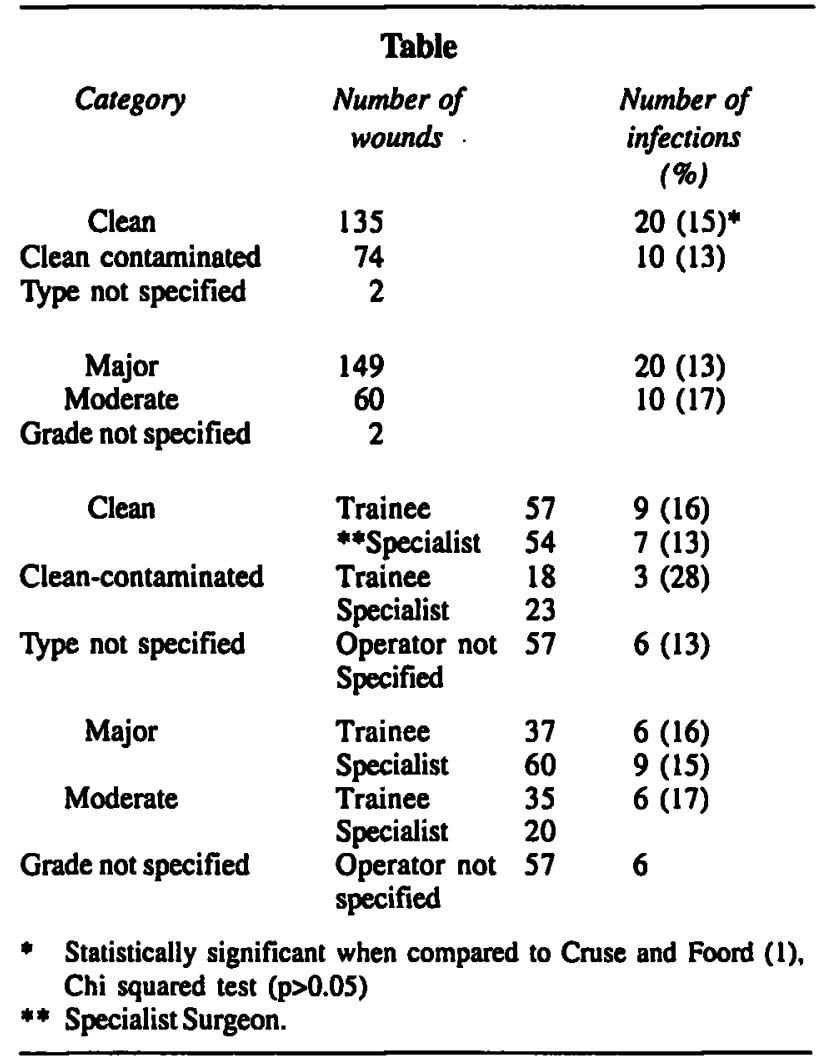

An important finding in our analysis was that the infection rate was high when a trainee surgeon performed the operation.

Drains were used in 124 wounds and there was a 17\% infection rate. When drains were not used in the remaining 87 operations, there was a $10 \%$ infection rate.

When there was renal dysfunction, ( 8 patients) the wound infection was $25 \%$, compared to the remaining 203 where the infection rate was $14 \%$.

Cruse and Foord reported one of the largest, prospective studies in 1980 (1). The infection rates of this study are used as reference values in our study.

Our wound infection rates are higher than those reported from other centers $(1,2)$. Cruse and Foord reported a clean surgery infection rate of 1.5 percent, but in our series the rate was 15 percent. In our theatres sterilised packs are not used for each operation. Instruments and towels are taken as required from an exposed trolley containing sterilised towels and instruments. Gloves are reused after washing and sterilising. Repeated use weakens the gloves and may make them porous. Our theatres do not have laminar flow systems and, being a teaching hospital, the presence of many people (medical students) in the operating rooms may increase wound contamination. The cost for each surgery is difficult to evaluate. In Sri Lanka, the annual cost to the state for each person is approximately $\$ 10$ (Rs 600$)$. In the UK the NHS spends approximately $\$ 900$. The above reasons may explain our higher infection rates for clean surgery.

Our clean-contaminated operations had an infection rate of $13 \%$ and this compares favourably with reported figures (1). This may be as a result of higher number of clean-contaminated operations performed by specialists (13\%) compared to the trainees rate of $28 \%$.

Major operations show a lower infection rate than moderate surgery and this may be due to specialists performing most major surgery. Trainees carried out more moderate surgery with higher infection rates.

There was a higher infection rate with the use of drains and this has been shown in other reports. The commonest drain used in our study is the corrugated rubber drain, and this can lead to retrograde bacterial flow, which may increase the infection rate. Patients with renal disease show an increased wound infection rate when compared to patients without renal disease.

Difficulties in collecting data even in prospective studies are documented (5). Our study shows an inadequacy in patient record keeping. Regular prospective audit of records can improve this.

In summary, we have a high infection rate for clean surgery and need to reduce this. Trainee surgeons should be made more aware of the need to reduce wound infection rates. Drains can lead to increased wound infection. Patients with renal impairment have higher wound infection rates. 
We are grateful to Professor A P R Aluwihare for help with the study and his scientific support. We also received valuable assistance from Dr. S Sivayogam (Senior Lecturer, Community Medicine, University of Sri Jayawardenapura) and Miss Amali De Silva (Statistician, University of Sri Jayawardenapura) for statistical analysis.

\section{References}

1. Cruse P J E, Foord R. The epidemiology of wound infection; a 10 year prospective study of 62939 wounds. Surgical Clinics of North America 1980; 60: 27-40.
2. Sawor R G, Pruett T L. Wound infections. Surgical Clinics of North America 1994; 74: 519-36.

3. Hunt M N, Chan A Y C, Karran S J. Post-operative complications, how much do they cost? Annals of the Royal College of Surgeons of England 1986; 68: 199-202.

4. Bailey IS, Karan S E, Toyn K, Brough P, Ranaboldo C, Karan $S \mathrm{~J}$. Community surveillance of complications after hemia surgery. British Medical Joumal 1992; 304: 469-71.

5. Mead P B, Pories S E, Hall P, Vacek PM, Davis S H, Gamelli R L. Decreasing the incidence of surgical wound infection. Archives of Surgery 1986; 122: 199-202.

E Rajasegaram, Senior Registrar, C Wijeratne, Research Medical Officer, R Mathangaweera, Nursing Officer, and S T Esufali, Senior Lecturer, University Surgical Unit, Teaching Hospital, Peradeniya. 